\title{
Nur wer die Milbe kennt, weiß sie zu vermeiden!
}

W illem Storm van Leeuwen (1882-1933) fuhr 1923 mit drei Patienten, die unter einem ganzjährigem Asthma litten, nach St. Moritz in die Schweiz. Sie waren hier sofort beschwerdefrei. Van Leeuwen hatte aber eine Büchse bei sich, gefüllt mit Hausstaub aus den Wohnungen der drei holländischen $\mathrm{Pa}$ tienten. Als er seine Patienten in St. Moritz nach eingetretener Beschwerdefreiheit aus dieser Büchse Staub einatmen ließ, hatten sie sofort wieder einen Asthmaanfall. Van Leeuwen hat über diesen bemerkenswerten historischen Provokationstest berichtet (Proc R Soc Med 1923; 17: 19) und damit den Nachweis erbracht, dass in dem Staub Allergene waren, die bei diesen Patienten einen akuten Asthmaanfall auslösen konnten.

Die Frage war aber offen, was das Allergen im Hausstaub war. Diese Frage lösten R. Voorhorst und das Ehepaar Spieksma, die Staubproben untersuchten und nachwiesen, dass Milben die Hauptallergenquelle im Hausstaub sind (Allergie und Asthma 1964; 10: 329-34).

Van Leeuwen war es auch, der den Gedanken der Allergenkarenz realisierte: Er baute bis 1926 in Leiden etwa 20 Häuser mit einer ,allergenfreien Kammer" - durch das Einsaugen von Luft und der Reinigung durch einen Filter -, so dass das Schlafzimmer frei von Allergenen sein sollte.

Auf der Grundlage tieferer biologischer Kenntnisse konnten die Karenzmaßnahmen gegen Hausstaubmilben weiter entwickelt werden. Einer der besten Experten auf dem Gebiet der Milbenbiologie, Herr Jörg-Thomas Franz, fasst in dieser Ausgabe die akarologischen

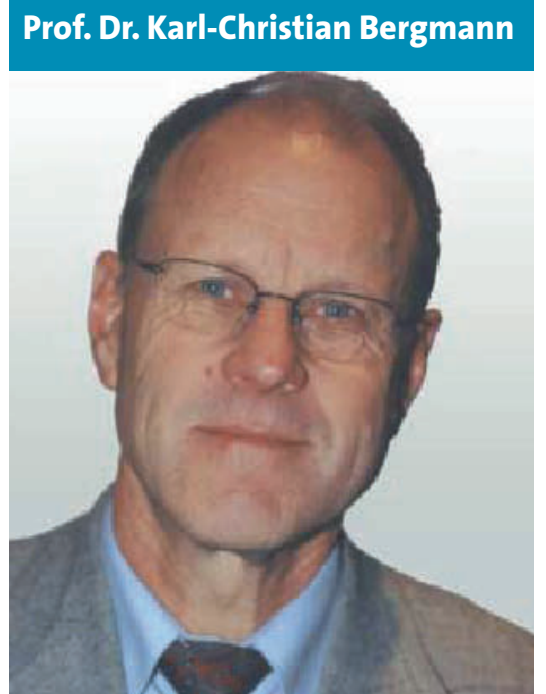

\section{Allergie- und Asthmaklinik Bad Lippspringe}

Grundlagen für Karenzmaßnahmen gegen Hausstaubmilben zusammen (Seite 443). Effektive Karenzmaßnahmen gegen Milben sind entscheidend von unserem Wissen um die Fortpflanzungsbiologie der Milben abhängig.

Noch vor den Hausstaubmilben waren es aber die sog. Vorratsmilben, die als Auslöser von Asthma vermutet wurden. Es war Dr. Herman Dekker aus dem Rheinland, der 1928 schrieb (Munch Med Wochenschr 1928; 75: 515-63): „Bei der mikroskopischen Untersuchung des Schlafzimmerstaubes [eines Kranken, der nur in seinem Schlafzimmer Asthma bekam] fand ich [...] Milben! Mein Erstaunen war groß, als ich bei systematischer Untersuchung [...] von Staub aus Matratzen [...] den Milbenbefall nicht als Ausnahme, sondern fast als Regel erheben musste. Meist fand ich Glycyphagus domesticus."
Horst Müsken fasst das aktuelle Wissen um Vorratsmilben und Möglichkeiten zur Karenz zusammen (Seite 452). Es wird deutlich, dass auch bei den Vorratsmilben die Kenntnis ihrer Biologie entscheidend ist für effektive Karenzmaßnahmen. Der Nachweis einer Vorratsmilbenallergie ist wichtig, er kann auf eine berufsbedingte Atemwegserkrankung hinweisen, und Vorratsmilbenallergene gehören in das Standardprogramm von Hauttestungen.

Schutz vor inhalierbaren Allergenen bieten Atemschutzgeräte. Dietrich Müller-Wening fast in einer Übersicht die derzeit angewendeten Atemschutzgeräte und das Wissen um ihre Wirksamkeit zusammen (Seite 460). Viele Landwirte können ihre Arbeit nur bei Benutzung von Atemschutzgeräten ausüben, so dass es sich offenbar um sehr wirksame Instrumente zur Allergenkarenz handelt.

Eine neue Leitlinie der DGAI zur erhöhten basalen Serumtryptasekonzentration oder Mastozytose als Risikofaktor einer Hymenopterengiftallergie erhebt die Forderung, bei Patienten mit einer Mastzellerkrankung die Insektengifthyposensibilisierung dauerhaft, ggf. auch lebenslang durchzuführen (Seite 440).

Angenehme Lektüre wünscht Ihnen

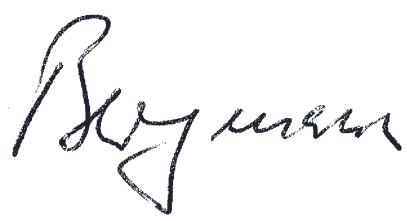

Prof. Dr. Karl-Christian Bergmann 